\title{
Integrable theories in any dimension: a perspective"
}

\author{
Orlando Alvarez $^{a}$, L.A. Ferreira ${ }^{b}$ and J. Sánchez Guillén ${ }^{c}$ \\ ${ }^{a}$ Department of Physics \\ University of Miami \\ P.O. Box 248046 \\ Coral Gables, FL 33124, USA \\ ${ }^{b}$ Instituto de Física Teórica - IFT/UNESP \\ Rua Pamplona 145 \\ 01405-900 São Paulo-SP, BRAZIL \\ ${ }^{c}$ Departamento de Física de Partículas, \\ Facultad de Física \\ Universidad de Santiago \\ E-15706 Santiago de Compostela, SPAIN
}

\begin{abstract}
We review the developments of a recently proposed approach to study integrable theories in any dimension. The basic idea consists in generalizing the zero curvature representation for two-dimensional integrable models to space-times of dimension $d+1$ by the introduction of a $d$-form connection. The method has been used to study several theories of physical interest, like self-dual Yang-Mills theories, Bogomolny equations, non-linear sigma models and Skyrme-type models. The local version of the generalized zero curvature involves a Lie algebra and a representation of it, leading to a number of conservation laws equal to the dimension of that representation. We discuss the conditions a given theory has to satisfy in order for its associated zero curvature to admit an infinite dimensional (reducible) representation. We also present the theory in the more abstract setting of the space of loops, which gives a deeper understanding and a more simple formulation of integrability in any dimension.
\end{abstract}

\footnotetext{
${ }^{1}$ Invited talk at the Meeting Trends in Theoretical Physics II at Buenos Aires, December 1998.
} 


\section{Introduction}

This paper addresses the long standing problem of the generalization of the zero curvature in classical integrability, from two dimensions to theories defined on space-times of any dimension. It consists of a review of the ideas proposed in [1] to implement that generalization, and also of the subsequent developments.

Integrability and, in a more general sense, construction of solutions and constants of motion, are important issues, given the relevance of nonperturbative aspects of field theories in High Energy Physics, Statistical Mechanics, Solid State Physics and Gravity.

In $2 d$, an impressive understanding of integrable theories has been achieved, which can be essentially encoded in the zero curvature formulation. In fact, the equations of motion of such theories, including relativistic invariant ones, can be naturally expressed in terms of a flat connection as $\left[\partial_{\nu}-A_{\nu}, \partial_{\mu}-A_{\mu}\right]=0$. The simplest (abelian) example is that of a free scalar field $\phi$, with $A_{\mu}=-\epsilon_{\mu \nu} \partial^{\mu} \phi$. The equations of motion of the Sine-Gordon models and its generalizations, the so called Affine Toda systems, are also expressed as zero curvature with $A_{\mu}$ taking values in an affine algebra [2]. One of the remarkable facts is that the algebraic structure involved in the zero curvature does not describe, in general, any explicit symmetry of the original equations of motion. In the case of theories presenting soliton solutions, a great unification is achieved in the framework of affine algebras, where there exists algebraic equivalent methods, like dressing of vacuum solutions or solitonic specialization giving the interesting solitonic solutions in a systematic and simple way [3].

The zero curvature formulation also provides a way of constructing conserved charges. This is easily seen choosing a finite two dimensional space-time with periodic boundary conditions on the time component of the connection. By Stokes calculus, any power $N$ of the $\operatorname{Tr}\left(P \exp \left(\int_{-L}^{L} A_{x}(x, t) d x\right)\right)^{N}$ is conserved in the time evolution (the path ordering $P$ is for the non-abelian general case). Despite the great advances in $2 d$ classical integrability, and also on some relations between integrability in parameter space and solutions of supersymmetric gauge theories in $4 d$ [4], very little was done in generalizing this beautiful picture to any dimension in space time, and understand directly those solutions in terms of generalized soliton dynamics, most interesting by itself. But expressing relativistic invariant field equations, known and possibly new ones, in such geometrical way which yields their integrals of motion, cannot be simple in higher dimensions. First of all, relativistic invariance requires higher rank tensors and connections, in principle with complicated gauge transformations. Besides, in $2 d$ 
the Lorentz transformations are rather trivial $x_{ \pm} \rightarrow \lambda^{ \pm 1} x_{ \pm}$and they can be imitated by the grading operator in the algebra, just making the forward (backward) light-cone to be positive (negative), incorporating also holomorphicity directly.

The main difficulties in extending of the integrability concepts to higher dimensions are associated to non-locality issues that rise when dealing with higher rank connections. Those problems can be circunvented by the introduction of auxiliary connections that allow for parallel transport. In addtion, once the invariant equations have been formulated, to get their integrals of motion one needs a generalization of Stokes calculus, which is directly related to the first problem of the transformation of the generalized connections. In fact Stokes formulae are problematic for the relevant non-abelian case even in $2 d$ [5]. Therefore, the starting point of [1] was to obtain a simple local expression for the non-abelian Stokes theorem, as we review in section 2 .

Our approach to generalize the zero curvature is based on the observation that the conservation laws can be expressed as the condition for the invariance of some quantity under the deformation of hypersurfaces. We are in fact constructing higher dimensional non-abelian Gauss-type laws. The main difficult is related to the implementation of the calculus of the variation of the hypersurfaces and the requirement that at the end one wants local conservation laws. The key observation for implementing the calculus was based on the formula satisfied by the Wilson loop $W_{c}$, calculated on a closed countour $C$, i.e.

$$
\frac{d W_{c}}{d t}=W_{c} T(F, 2 \pi, t)
$$

where $t$ parametrizes the variations of the countour that keep a given fixed point $x_{0}$ fixed, and

$$
T(F, 2 \pi, t) \equiv \int_{0}^{2 \pi} d \sigma W^{-1} F_{\mu \nu} W \frac{d x^{\mu}}{d \sigma} \frac{d x^{\nu}}{d t}
$$

and $F_{\mu \nu}$ is the curvature of the connection $A_{\mu}$ defining the Wilson line $W$, i.e.

$$
\frac{d W}{d \sigma}=-A_{\mu} \frac{d x^{\mu}}{d \sigma} W
$$

and the integral in $T$ is along the curve parameter $\sigma$. Eq.(1.1), which is easily obtained from Interaction Picture method in [1], expresses loop independence when the connection is flat and it is essentially non-local. This simple formulation gives the idea for the zero curvature generalization to $3 d$ : introduce a functional of the fields $V$ defined by its variation under deformations of the loops through the equation

$$
\frac{d V}{d t}=V T(B, 2 \pi, t)
$$

where $B_{\mu \nu}$ is a general antisymmetric tensor functional and

$$
T(B, 2 \pi, t) \equiv \int_{0}^{2 \pi} d \sigma W^{-1} B_{\mu \nu} W \frac{d x^{\mu}}{d \sigma} \frac{d x^{\nu}}{d t},
$$


One looks then for the conditions for $V$, now a surface integral, to be independent of the surface scanned by the different loops. Notice that the integrand in $T(B, 2 \pi, t)$ is a local function. The form of $T$ will be essential to guarantee Lorentz and gauge invariant time evolution. Generalizing directly the previous 2d Interaction picture computation varying now with respect to a new orthogonal parameter $\tau$, we obtain a rather complicated expression, albeit with a transparent geometrical and physical meaning. It is a generalized non-abelian Gauss law and, as shown in detail in section 2.1, it corresponds to parallel transport in the space of loops. This geometrical theory tell us how to proceed in any dimension and makes very natural the translation of the surface independence to different local equations of motion and/or integrability conditions, which are deduced directly in section 3. A natural condition is the flatness of the first connection $F_{A}=0$, which guarantees independence of how the surface is scanned by loops, and then a sufficient condition for the vanishing of the non-local curvature associated to $V$ is the constant covariance of the tensor connection $D_{A} B=0$. This nesting of constant covariance and vanishing of the commutator of two $d-$ forms and flatness of the lower $d-1, d-2 \ldots$ curvatures (or possibly some of them) is generic and it is worked out in detail also for the $4 d$ (see [1] for details).

The Lorentz covariant and gauge invariant formalism is very general and one has lots of algebraic and topological structures to explore which support those local formulations and which will yield new formulations of known theories or new ones, with a systematic method to get their integrals of motion and solutions. In $3 d$, the most simple case is choosing $B_{\mu \nu}(0)$ on an abelian subalgebra. Notice that $B_{\mu \nu}$ can then be uniquely defined at any point $x$ by parallel transport under $A_{\mu}$. The integrability conditions in this case correspond to the BF theory, Chern-Simons and other "topological" theories in 3d. That the construction works for these topological cases is reassuring and one can define now naturally new observables like invariants for link crossings or study topological defects, but the importance of conserved quantities is, of course, with time evolution. On the other hand very little is clearly understood about non-perturbative solutions and classical integrability for the situation when there is full relativistic dynamics in dimension higher than 2. So we concentrated in [1] on the simplest genuine $2+1$ theory, the $O(3)$ nonlinear model, although most of the approach can be used for a general sigma model as shown later in [6, 7]. In the four dimensional case, it was shown in [1] how to express self-dual Yang-Mills and BPS theory in such zero curvature formulation of local integrability conditions for volume independence. We obtain also a reduction of the equations which exhibit directly their conservation character.

One of the interesting aspects of [1] is that many theories presenting the local zero curvature are not integrable in the sense of possessing an infinite number of conservation 
laws. However, some of those theories contain integrable submodels that do present an infinite number of conserved currents. We discuss in section the conditions a given theory has to satisfy to present an infinity number of conserved local currents. The main point is that its zero curvature representation has to involve infinite dimensional representations of a Lie algebra, or equivalently as we explain, and infinite dimensional non-semisimple Lie algebra of the Poincaré type.

\section{Geometrical approach to integrability}

The zero curvature condition in two dimensional spacetime, know as the ZakharovShabat equation 8 is given by

$$
F_{\mu \nu} \equiv\left[\partial_{\mu}+A_{\mu}, \partial_{\nu}+A_{\nu}\right]=0 \quad \mu, \nu=0,1
$$

One of the consequences of it is that it leads to conservation laws. In order to see that, consider a quantity $W$ defined by paralell transport with a connection $A_{\mu}$,

$$
\frac{d W}{d \sigma}+A_{\mu} \frac{d x^{\mu}}{d \sigma} W=0
$$

Consider now a closed curve $\Gamma$, and let $\Sigma$ be a two dimensional surface having $\Gamma$ as its boundary. The nonabelian Stokes theorem states that the quantity $W$ can be determined either by integrating (2.2) on $\Gamma$ or on $\Sigma$. More precisely one has

$$
W(\Gamma)=P \exp \left(\int_{\Gamma} d \sigma A_{\mu} \frac{d x^{\mu}}{d \sigma}\right)=\mathcal{P} \exp \left(\int_{\Sigma} d \tau d \sigma W^{-1} F_{\mu \nu} W \frac{d x^{\mu}}{d \sigma} \frac{d x^{\nu}}{d \tau}\right)
$$

where $P$ and $\mathcal{P}$ mean path and surface ordering respectively (see [1] for details).

Therefore, if the connection $A_{\mu}$ is flat, i.e. (2.1) holds true, then $W$ is equal to unity for closed loops. Then, conserved quantities are constructed as follows. First we consider the case where spacetime is a cylinder. At a fixed time $t_{0}$ consider a loop $\gamma_{0}$ beginning and ending at $x_{0}$. At a later fixed time $t_{1}$ consider a loop $\gamma_{1}$ also beginning and ending at $x_{0}$. Let $\gamma_{01}$ be a path connecting $\left(t_{0}, x_{0}\right)$ with $\left(t_{1}, x_{0}\right)$. The flat connection allows us to integrate the parallel transport equation (2.2) along two different paths obtaining $W\left(\gamma_{0}\right)=W\left(\gamma_{01}\right)^{-1} W\left(\gamma_{1}\right) W\left(\gamma_{01}\right)$. We first observe that $W\left(\gamma_{0}\right)$ transforms under a gauge transformation $g(x)$ as $W\left(\gamma_{0}\right) \rightarrow g\left(x_{0}\right) W\left(\gamma_{0}\right) g\left(x_{0}\right)^{-1}$. The conserved quantity should be gauge invariant. If $\chi$ is a character for the group $G$ we have that $\chi\left(W\left(\gamma_{0}\right)\right)$ will be gauge invariant. Also $\chi\left(W\left(\gamma_{0}\right)\right)=\chi\left(W\left(\gamma_{1}\right)\right)$. Thus we can construct a conserved gauge invariant quantity

$$
\chi\left(W\left(\gamma_{0}\right)\right)
$$


for every independent character of the group. These are the constants of motion in the zero curvature construction. Note that the data needed to compute $\chi\left(W\left(\gamma_{0}\right)\right)$ is all determined at time $t_{0}$.

In the case where the spacetime is two dimensional Minkowski space one has to impose physically sensible boundary conditions at spatial infinity. Note that $P \exp \left(\int_{-\infty}^{\infty} A_{x} d x\right)$ is not gauge invariant if one allows nontrivial gauge transformations at infinity. In setting up the problem one has to choose the correct physical boundary conditions which may for example require that $A_{0}$ vanishes at infinity. Depending on the details one can construct a conserved quantity by a slight modification of the construction above.

The conserved quantities (2.4) are nonlocal because in general the connection $A_{\mu}$ lies in a nonabelian algebra. However, in cases like the affine Toda models [2], it is possible to get local conservation laws by gauge transforming $A_{\mu}$ into an abelian subalgebra.

The basic idea in [四] to bring such concepts to higher dimensions, is to introduce quantities integrated over hypersurfaces and to find the conditions for them to be independent of deformations of the hypersurfaces which keep their boundaries fixed. Such an approach will certainly lead to conservation laws in a manner very similar to the two dimensional case. However, the main problem of that it is how to introduce nonlinear zero curvatures keeping things as local as possible. The way out is to introduce auxiliary connections to allow for parallel transport. The number of possibilities of implementing those ideas increase with the dimensionality of space-time. However, the simplest scenario is that where, in a space-time of dimension $d+1$, one introduces a rank $d$ antisymmetric tensor $B_{\mu_{1} \mu_{2} \ldots \mu_{d}}$ and a vector $A_{\mu}$. The idea can perhaps be best stated using a formulation in "loop space". On a $d+1$ dimensional space-time $M$ one considers the space $\Omega^{d-1}\left(M, x_{0}\right)$ of $d-1$ dimensional closed hypersurfaces based at a fixed point $x_{0} \in M$. One then introduces on such "higher loop space" a 1-form $\mathcal{A}$ which is basically the quantity $W^{-1} B_{\mu_{1} \mu_{2} \ldots \mu_{d}} W$ integrated over the closed hypersurfaces (see [1] for details). The quantity $W$ is defined in terms of the vector $A_{\mu}$ through (2.2). However, for $W$ to be independent of the way one integrates it from $x_{0}$ to a given point on the hypersurface, one has to assume that $A_{\mu}$ is flat, i.e.

$$
F_{\mu \nu}=\left[D_{\mu}, D_{\nu}\right]=\partial_{\mu} A_{\nu}-\partial_{\nu} A_{\mu}+\left[A_{\mu}, A_{\nu}\right]=0 ; \quad \mu, \nu=0,1,2 \ldots d
$$

with

$$
D_{\mu} \cdot \equiv \partial_{\mu} \cdot+\left[A_{\mu}, \cdot\right]
$$

Roughly speaking a $d$ dimensional closed hypersurface in $M$, based at $x_{0}$, corre- 
sponds to a (one dimensional) loop in $\Omega^{d-1}\left(M, x_{0}\right)$. Therefore, the condition to have things independent of deformation of hypersurfaces translates in such "higher loop space" to the zero curvature condition for $\mathcal{A}$, namely

$$
\mathcal{F}=\delta \mathcal{A}+\mathcal{A} \wedge \mathcal{A}=0
$$

The relation (2.7) (together with (2.5)) is the generalization of the zero curvature (2.1) to higher dimensions proposed in [1].

The idea of constructing conserved quantities using (2.7) is the same as the two dimensional case decribed above. Indeed, the condition (2.7) implies that the path ordered exponential of $\mathcal{A}$ along a closed loop in the higher loop space $\Omega^{d-1}\left(M, x_{0}\right)$ is independent of the loop. Following arguments similar to those below (2.3), one sees that the conserved quantities are path ordered exponentials of $\mathcal{A}$ along closed loop at a given fixed time. Since loops in $\Omega^{d-1}\left(M, x_{0}\right)$ correspond to $d$-dimesnional closed surfaces in the $d+1$-dimensional spcaetime $M$, one gets that such quantities are indeed integrated over the physical space. Although the analogy with the two dimensional case looks straitghforward, the implementation is quite involved due to the ordering of the integration, the respect to gauge invariance and boundary conditions. We refer to [1] for those very important explanations.

In order to make the formulas more explicit we give below the discussion of the $2+1$ dimensional case.

\subsection{The zero curvature in three dimensions}

Assume we have a principal bundle $P \rightarrow M$ with connection. For a fixed point $x_{0} \in M$ let $\Omega\left(M, x_{0}\right)$ be the space of all loops based at $x_{0}$ :

$$
\Omega\left(M, x_{0}\right)=\left\{\gamma: S^{1} \rightarrow M \mid \gamma(0)=x_{0}\right\}
$$

we now want to construct a principal $G$-bundle over $\Omega\left(M, x_{0}\right)$ with connection. Note that the structure group of the bundle is a finite dimensional group not a loop group. It will be the trivial bundle $\mathcal{P}=\Omega\left(M, x_{0}\right) \times G$. Conceptually the bundle is constructed as follows. Over $x_{0} \in M$ the bundle $P \rightarrow M$ has fiber $P_{x_{0}}$ which is isomorphic to $G$. All loops in $\Omega\left(M, x_{0}\right)$ have $x_{0} \in M$ as a starting point so we can consider them having $P_{x_{0}}$ in common. This is the common fiber in the cartesian product $\Omega\left(M, x_{0}\right) \times G$. Mathematically we have a natural map $\pi: \Omega\left(M, x_{0}\right) \rightarrow M$ given by $\pi(\gamma)=x_{0}$. The bundle $\mathcal{P}$ is just the pullback bundle $\pi^{*} P$, see [9]. Since $\mathcal{P} \rightarrow \Omega\left(M, x_{0}\right)$ is a trivial bundle we can put the trivial connection on it. There is a more interesting connection 
one can put on it which exploits the connection on the bundle $P \rightarrow M$. Consider a Lie algebra valued 2-form $B$ on $M$ such that under the transition function $\phi$ we have $B \rightarrow \phi^{-1} B \phi$. Let $W(\sigma)$ be the parallel transport operator from the point $x(0)=x_{0}$ to the point $x(\sigma)$ along the loop $\gamma$. We can assign the Lie algebra valued 1-form

$$
\mathcal{A}[x(\sigma)]=\int_{0}^{2 \pi} d \sigma W(\sigma)^{-1} B_{\mu \nu}(x(\sigma)) W(\sigma) \frac{d x^{\mu}}{d \sigma} \delta x^{\nu}(\sigma)
$$

The transformation laws of the above are determined by $\phi\left(x_{0}\right)$ which is clearly associated with the common fiber $P_{x_{0}}$. Thus we can define a connection on $\mathcal{P}$ by

$$
\varpi=-d g g^{-1}+g\left(\int_{0}^{2 \pi} d \sigma W(\sigma)^{-1} B_{\mu \nu}(x(\sigma)) W(\sigma) \frac{d x^{\mu}}{d \sigma} \delta x^{\nu}(\sigma)\right) g^{-1} .
$$

Thus we can treat $\mathcal{A}$ as the connection in a certain trivialization. The curvature is given by $\mathcal{F}=\delta \mathcal{A}+\mathcal{A} \wedge \mathcal{A}$.

What do we mean when we say that we want to have parallel transport independent of path in $\Omega\left(M, x_{0}\right)$ ? Look at the space $\Omega\left(M, x_{0}\right)$ and consider a curve $\Gamma$ in $\Omega\left(M, x_{0}\right)$ parametrized by $\tau$ such that $\Gamma(0)$ is the "constant curve" $x_{0}$. Note that for fixed $\tau, \Gamma(\tau)$ is a curve $x_{\tau}(\sigma)$ for $\sigma \in[0,2 \pi]$ in $M$. Thus it is convenient to "write" $\Gamma$ as $x(\sigma, \tau)$. The statement that parallel transport be independent of the choice of curve $\Gamma \in \Omega\left(M, x_{0}\right)$ with fixed starting and ending points is the statement that the curvature vanish. If one wants the parallel transport between points in $\Omega\left(M, x_{0}\right)$ to be independent of path then parallel transport should be path independent in $M$. The reason for this is that a loop in $\Omega\left(M, x_{0}\right)$ beginning at the trivial loop may be viewed as a map from the square $[0,2 \pi]^{2}$ to $M$ such that $\partial[0,2 \pi]^{2}$ gets mapped to $x_{0}$. To get the same result for two different sets of "constant" $\tau$ curves associated with the same closed 2-submanifold in $M$, one needs ordinary parallel transport to be path independent, i.e. $F=0$.

In order to perform the curvature computation we need the standard result:

$$
\begin{aligned}
W(\sigma)^{-1} \delta W(\sigma) & =-W(\sigma)^{-1} A_{\mu}(x(\sigma)) W(\sigma) \delta x^{\mu}(\sigma) \\
& +\int_{0}^{\sigma} d \sigma^{\prime} W\left(\sigma^{\prime}\right)^{-1} F_{\mu \nu}\left(x\left(\sigma^{\prime}\right)\right) W\left(\sigma^{\prime}\right) \frac{d x^{\mu}}{d \sigma^{\prime}} \delta x^{\nu}\left(\sigma^{\prime}\right) .
\end{aligned}
$$

We also need definition (2.9). Let $\delta$ be the exterior derivative on the space $\Omega\left(M, x_{0}\right)$ and thus $\delta^{2}=0$ and

$$
\delta x^{\mu}(\sigma) \wedge \delta x^{\nu}\left(\sigma^{\prime}\right)=-\delta x^{\nu}\left(\sigma^{\prime}\right) \wedge \delta x^{\mu}(\sigma)
$$

Computing the curvature $\mathcal{F}=\delta \mathcal{A}+\mathcal{A} \wedge \mathcal{A}$ is tedious and we refer to [1] for the details. The result is

$$
\mathcal{F}=-\frac{1}{2} \int_{0}^{2 \pi} d \sigma W(\sigma)^{-1}\left[D_{\lambda} B_{\mu \nu}+D_{\mu} B_{\nu \lambda}+D_{\nu} B_{\lambda \mu}\right](x(\sigma)) W(\sigma)
$$




$$
\begin{gathered}
\times \frac{d x^{\lambda}}{d \sigma} \delta x^{\mu}(\sigma) \wedge \delta x^{\nu}(\sigma) \\
-\int_{0}^{2 \pi} d \sigma \int_{0}^{\sigma} d \sigma^{\prime}\left[F_{\kappa \mu}^{W}\left(x\left(\sigma^{\prime}\right)\right), B_{\lambda \nu}^{W}(x(\sigma))\right] \frac{d x^{\kappa}}{d \sigma^{\prime}} \frac{d x^{\lambda}}{d \sigma} \delta x^{\mu}\left(\sigma^{\prime}\right) \wedge \delta x^{\nu}(\sigma) \\
+\frac{1}{2} \int_{0}^{2 \pi} d \sigma \int_{0}^{2 \pi} d \sigma^{\prime}\left[B_{\kappa \mu}^{W}\left(x\left(\sigma^{\prime}\right)\right), B_{\lambda \nu}^{W}(x(\sigma))\right] \frac{d x^{\kappa}}{d \sigma^{\prime}} \frac{d x^{\lambda}}{d \sigma} \delta x^{\mu}\left(\sigma^{\prime}\right) \wedge \delta x^{\nu}(\sigma) .
\end{gathered}
$$

The vanishing of $\mathcal{F}$, i.e. relation $(2.7)$, is our generalization of the zero curvature to higher dimensions. It implies hypersurface indepence and conservation laws as we explained above.

\section{Local integrability conditions}

The condition (2.7) that the loop space curvature should vanish is local in $\Omega^{d-1}\left(M, x_{0}\right)$, but it is highly non-local in the spacetime $M$. We now discuss some sufficient local conditions for the vanishing of $(2.10)$.

Let $\mathcal{G}$ be a Lie algebra and $R$ be a representation of it. We introduce the nonsemisimple Lie algebra $\mathcal{G}_{R}$ as

$$
\begin{aligned}
& {\left[T_{a}, T_{b}\right]=f_{a b}^{c} T_{c}} \\
& {\left[T_{a}, P_{i}\right]=P_{j} R_{j i}\left(T_{a}\right)} \\
& {\left[P_{i}, P_{j}\right]=0}
\end{aligned}
$$

where $T_{a}$ constitute a basis of $\mathcal{G}$ and $P_{i}$ a basis for the abelian ideal $P$ (representation space). The fact that $R$ is a matrix representation, i.e.

$$
\left[R\left(T_{a}\right), R\left(T_{b}\right)\right]=R\left(\left[T_{a}, T_{b}\right]\right)
$$

follows from the Jacobi identities.

We take the connection $A_{\mu}$ to be in $\mathcal{G}$ and the rank $d$ antisymmetric tensor $B_{\mu_{1} \mu_{2} \ldots \mu_{d}}$ to be in $P$, i.e.

$$
A_{\mu}=A_{\mu}^{a} T_{a}, \quad B_{\mu_{1} \mu_{2} \ldots \mu_{d}}=B_{\mu_{1} \mu_{2} \ldots \mu_{d}}^{i} P_{i}
$$

Then a set of sufficient local conditions for the vanishing of the curvature $\mathcal{F}$ in (2.7) is given by

$$
D_{\mu} \tilde{B}^{\mu}=0 ; \quad F_{\mu \nu}=0
$$

where we have introduced the dual of $B_{\mu_{1} \mu_{2} \ldots \mu_{d}}$ as

$$
\tilde{B}^{\mu} \equiv \frac{1}{d !} \varepsilon^{\mu \mu_{1} \mu_{2} \ldots \mu_{d}} B_{\mu_{1} \mu_{2} \ldots \mu_{d}}
$$


Indeed, in the $2+1$ dimensional case the conditions (3.1) and (3.3) imply that

$$
W^{-1} B_{\mu \nu} W \in P
$$

and therefore the commutator in the last term of (2.10) vanishes, since $P$ is abelian. The first condition in (3.4) in the $2+1$ dimensional case reads

$$
D_{\lambda} B_{\mu \nu}+D_{\mu} B_{\nu \lambda}+D_{\nu} B_{\lambda \mu}=0
$$

Therefore, together with $F_{\mu \nu}=0$, it implies that the remaining terms of (2.10) vanish too.

The relations (3.4) are the local integrability conditions which we introduce for theories defined on a spacetime of any dimension, and which constitutes a generalization of the zero curvature condition (2.1) in two dimensions. They lead to local conservation laws. Indeed, since the connection $A_{\mu}$ is flat it can be written as

$$
A_{\mu}=-\partial_{\mu} W W^{-1}
$$

and consequently (3.4) imply that the currents

$$
J_{\mu} \equiv W^{-1} \tilde{B}_{\mu} W
$$

are conserved

$$
\partial_{\mu} J^{\mu}=0
$$

The zero curvature conditions (3.4) are invariant under the gauge transformations

$$
\begin{aligned}
& A_{\mu} \rightarrow g A_{\mu} g^{-1}-\partial_{\mu} g g^{-1} \\
& \tilde{B}_{\mu} \rightarrow g \tilde{B}_{\mu} g^{-1}
\end{aligned}
$$

and

$$
\begin{aligned}
& A_{\mu} \rightarrow A_{\mu} \\
& \tilde{B}_{\mu} \rightarrow \tilde{B}_{\mu}+\varepsilon_{\mu \mu_{1} \ldots \mu_{d}} D^{\mu_{1}} \alpha^{\mu_{2} \ldots \mu_{d}} \equiv \tilde{B}_{\mu}+D^{\nu} \tilde{\alpha}_{\mu \nu}
\end{aligned}
$$

where we have introduced the dual $\tilde{\alpha}_{\mu \nu} \equiv \varepsilon_{\mu \nu \mu_{2} \ldots \mu_{d}} \alpha^{\mu_{2} \ldots \mu_{d}}$. In (3.11) $g$ is an element of the group obtained by exponentiating the Lie algebra $\mathcal{G}$. The transformations (3.12) are symmetries of (3.4) as a consequence of the fact that the connection $A_{\mu}$ is flat, i.e. $\left[D_{\mu}, D_{\nu}\right]=0$. In addition, the parameters $\alpha^{\mu_{1} \ldots \mu_{d-1}}$ take values in the abelian ideal $P$.

The currents (3.9) are invariant under the transformations (3.11), but under (3.12) they transform as

$$
J_{\mu} \rightarrow J_{\mu}+\varepsilon_{\mu \mu_{1} \ldots \mu_{d}} \partial^{\mu_{1}}\left(W^{-1} \alpha^{\mu_{2} \ldots \mu_{d}} W\right)=J_{\mu}+\partial^{\nu}\left(W^{-1} \tilde{\alpha}_{\mu \nu} W\right)
$$


The transformations (3.11) and (3.12) do not commute and their algebra is isomorphic to the non-semisimple algebra $\mathcal{G}_{R}$ introduced in (3.1). The nontrivial gauge transformations allow in principle the dressing of vacuum solutions to obtain general ones, as discussed in [1].

\section{Integrable submodels}

The number of conserved currents one gets from (3.9) is equal to the dimension of the representation of $\mathcal{G}$, defined by the generators $P_{i}$ of $\mathcal{G}_{R}$. Consequently, the notion of integrability in such approach is related to infinite dimensional representations, or equivalently to infinite dimensional non-semisimple Lie algebras of the type (3.1). That is similar to the two dimensional case where the appearence of infinte number of charges is also linked to infinite dimensional Lie algebras. However, those are in general of the affine type, and it is now quite well understood the role they have in soliton theory and exact methods of construction of solutions. In our approach, the role of algebraic structures like (3.1) is not fully understood yet, but we believe it must have profound consequences in the study of higher dimensional integrable theories.

One point that became clear recently is that of integrable submodels. It is known that conditions like self-duality in Yang-Mills theories and the Bogomolny equations in gauge theories with symmetry spontaneously broken by a Higgs in the adjoint representation, play a crucial role in the construction of submodels which possess properties of solvability not present in the full theory. Those conditions lead to a saturation of a bound on the Euclidean action in the case of self-dual Yang-Mills and on the energy in the case of Bogomolny equations.

In our approach a similar thing happens, however involving quite different structures. It does lead to conditions for integrable submodels, but apparently not to saturation of bounds. In the examples studied so far what one gets is the following. Suppose that using the gauge symmetries (3.11) and (3.12) one can find a gauge where the connection $A_{\mu}$ can be split as [6]

$$
A_{\mu}=A_{\mu}^{S}+A_{\mu}^{K}
$$

where $A_{\mu}^{S / K}$ are the components of $A_{\mu}$ in the decomposition

$$
\mathcal{G}=S+K
$$

with $K$ being a subalgebra of $\mathcal{G}$, and $S$ its complement in $\mathcal{G}$. Suppose in addition that

$$
\left[A_{\mu}^{S}, \tilde{B}^{\mu}\right]=0
$$


Then the first zero curvature (3.4) becomes

$$
\partial^{\mu} \tilde{B}_{\mu}+\left[A^{\mu K}, \tilde{B}_{\mu}\right]=\left(\partial^{\mu} \tilde{B}_{\mu, i}+A_{a}^{\mu K} \tilde{B}_{\mu, j} R\left(K_{a}\right)_{i j}\right) P_{i}
$$

where $A^{\mu K}=A_{a}^{\mu K} K_{a}$, with $K_{a}$ being the generators of $K$, and $R\left(K_{a}\right)_{i j}$ being the matrix representation of $K$ defined by the $P_{i}$ 's, i.e.

$$
\left[K_{a}, P_{i}\right]=P_{j} R\left(K_{a}\right)_{j i}
$$

Therefore, the zero curvature condition is only determined by the representation of $K$ defined by the subspace $P$. Such a representation is in general reducible, and we shall denote the branching as

$$
R=\sum_{l} R_{l}^{K}
$$

with $R_{l}^{K}$ being irreducible representations of $K$. Suppose now that a given representation $R^{\lambda}$ of $\mathcal{G}$ presents a branching rule

$$
R^{\lambda}=\sum_{l} R_{l}^{K}+\text { something }
$$

Then we can introduce an operator

$$
\tilde{B}_{\mu}^{\lambda} \equiv \tilde{B}_{\mu, i} P_{i}^{\lambda}
$$

where $\tilde{B}_{\mu, i}$ are the same coefficients as in the expansion of the original $\tilde{B}_{\mu}$ in terms of the basis $P_{i}$ of the representation $R$ of $\mathcal{G}$, i.e. $\tilde{B}_{\mu}=\tilde{B}_{\mu, i} P_{i}$. In addition, $P_{i}^{\lambda}$ are the generators of $\mathcal{G}_{R^{\lambda}}$ associated to the representation $R^{\lambda}$, corresponding to the subspace $\sum_{l} R_{l}^{K}$, and transforming exactly as the $P_{i}$ 's under the subalgebra $K$, i.e.

$$
\left[K_{a}, P_{i}^{\lambda}\right]=P_{j}^{\lambda} R\left(K_{a}\right)_{j i}
$$

Therefore, one gets that

$$
\begin{aligned}
D^{\mu} \tilde{B}_{\mu}^{\lambda} & =\partial^{\mu} \tilde{B}_{\mu}^{\lambda}+\left[A^{\mu}, \tilde{B}_{\mu}^{\lambda}\right] \\
& =\left(\partial^{\mu} \tilde{B}_{\mu, i}+A_{a}^{\mu K} \tilde{B}_{\mu, j} R\left(K_{a}\right)_{i j}\right) P_{i}^{\lambda} \\
& +\left[A^{\mu S}, \tilde{B}_{\mu}^{\lambda}\right]
\end{aligned}
$$

Notice that the first term after the last equality in (4.10) corresponds to the zero curvature for the theory under consideration, namely (4.4). Consequently, if one imposes the constraint

$$
\left[A^{\mu S}, \tilde{B}_{\mu}^{\lambda}\right]=A_{r}^{\mu S} \tilde{B}_{\mu}^{i}\left[S_{r}, P_{i}^{\lambda}\right]=0
$$


with $S_{r}$ being the generators of the subspace $S$, one gets a submodel with the conserved currents given by (see $(3.9))$

$$
J_{\mu}^{\lambda} \equiv W^{-1} \tilde{B}_{\mu}^{\lambda} W
$$

Suppose now that there exists an infinite number of representations, like $R^{\lambda}$, satisfying (4.7) such that the conditions (4.11) impose the same set of constraints on the model. Then the submodel defined by the equations (4.4) and the constraints (4.11) possesses an infinite number of local conserved currents. Several examples fullfilling those requirements were constructed in [1, 7, 6, 10].

An important case where the above construction works is when the representation of $\mathcal{G}$ defined by the $P_{i}$ 's, possesses at least one charge zero singlet of the subalgebra $K$, i.e. there exists in the abelian subalgebra $P$ a generator $P_{\Lambda}$, such that [6]

$$
\left[K_{a}, P_{\Lambda}\right]=0
$$

Then one can easily construct representations satisfying (4.7) by considering tensor products of the representation $R$, defined by the $P_{i}$ 's, with itself. The subsapces given by the tensor products of $R$ with the singlet $P_{\Lambda}$, transform like $\sum_{l} R_{l}^{K}$. For instance, in the case of $R \otimes R$ one has that $P_{\Lambda} \otimes R$ (equivalently $R \otimes P_{\Lambda}$ ) satisfies

$$
\left[1 \otimes K_{a}+K_{a} \otimes 1, P_{\Lambda} \otimes P_{i}\right]=P_{\Lambda} \otimes P_{j} R\left(K_{a}\right)_{j i}
$$

For the case of $(\otimes R)^{n}$ any representation of the form $\left(\otimes P_{\Lambda}\right)^{l} \otimes R\left(\otimes P_{\Lambda}\right)^{n-l-1}$ is equivalent to $R$ (viewed as representations of the subalgebra $K$ ). Therefore, one introduces the potentials

$$
\begin{aligned}
A_{\mu}^{(n)} & \equiv A_{\mu}^{\alpha} \sum_{l=0}^{n-1}(\otimes 1)^{l} \otimes T_{\alpha}(\otimes 1)^{n-l-1} \\
\tilde{B}_{\mu}^{(n)} & \equiv \tilde{B}_{\mu, i} \sum_{l=0}^{n-1} c_{n, l}\left(\otimes P_{\Lambda}\right)^{l} \otimes P_{i}\left(\otimes P_{\Lambda}\right)^{n-l-1}
\end{aligned}
$$

where we have denoted $A_{\mu}=A_{\mu}^{\alpha} T_{\alpha}$, with $T_{\alpha}$ being the generators of $\mathcal{G}$, and where $c_{n, l}$ are constants. We introduce such constants because one can rescale the basis of each irreducible component of the representations of $K$ independently, without affecting the equations (4.4). Only the constraints, defining the submodel, are affected by the constants $c_{n, l}$.

Notice that the curvature $F_{\mu \nu}^{(n)}$ associated to the connection $A_{\mu}^{(n)}$ vanishes as a consquence of the fact that $A_{\mu}$ is flat. Therefore, the first condition in (3.4) leads in this case, to the same equations as following equations as (4.4), i.e.

$$
\partial^{\mu} \tilde{B}_{\mu, i}+A_{a}^{\mu K} \tilde{B}_{\mu, j} R\left(K_{a}\right)_{i j}=0
$$


and the constraints

$$
A_{\mu}^{S, r} \tilde{B}_{i}^{\mu}\left[\left(\sum_{m=0}^{n-1}(\otimes 1)^{m} \otimes S_{r}(\otimes 1)^{n-m-1}\right),\left(\sum_{l=0}^{n-1} c_{n, l}\left(\otimes P_{\Lambda}\right)^{l} \otimes P_{j}\left(\otimes P_{\Lambda}\right)^{n-l-1}\right)\right]=0
$$

Therefore, since (4.16) are the same equations as (4.4) we have a submodel, and the subclass of solutions is determined by the constraints (4.17).

The conserved currents obtained from the zero curvature are (see (3.9))

$$
J_{\mu}^{\lambda(n)} \equiv\left(\otimes W^{-1}\right)^{n} \tilde{B}_{\mu}^{(n)}(\otimes W)^{n}
$$

Therefore, if we can choose the constants $c_{n, l}$ in such way that (4.17) imposes the same set of constraints for any $n$, then one gets that the corresponding submodel possesses an infinity number of conserved currents.

\section{Applications and Outlook}

The first applications of our zero curvature generalization have been to produce new geometrical formulations of well known theories in higher dimensions. The most obvious way to have flatness locally is by requiring each component of $B_{\mu \nu}$ to be covariantly constant and to commute at the same point. This simplest possibility, which has not been reviewd here, produces topological theories like Chern-Simons [11]. Since these theories are rather well understood in the quantum case, a possible application is to compare to the classical solution. Self dual Yang-Mills or BPS are also easily incorporated and were also given in [1] as $4 \mathrm{~d}$ examples.

The case which has been studied in more detail is when the local equation are based on a non-semisimple Lie algebra, which has produced many new results, specially obtaining reductions with infinite number of conserved currents. First it was applied to $C P^{1}$ in [1], and to the chiral $(s u(2))$ model in [7], later generalized to Grassmannian models in [12] and then to homogeneous spaces in general in [6], including symmetric spaces (compact and noncompact). In this last general formulation, new models and many classes of the previous cases where treated with new results and insights. In particular, the reason behind the infinity of conserved currents, discussed only heuristically in [1], has been fully understood in [6] with the coset construction $G / K$ in terms of the branching of representations of $G$ in those of $K$ with some singlets states playing a special and important role. This has been explained in detail here (see section $₫$ ) directly in terms of the decomposition of the algebra of $G$ in a subalgebra $K$ and its complement. 
One of the most relevant aspects of our approach is the reduction to submodels possessing infinite number of conserved charges. Although that may resemble the BPS condition, it apparently does not involve saturation of any bound. Such methods have also been applied to Skyrme and Skyrme-type models [10, 13]. The existence of solutions for the constraints is highly non trivial, specially for the hedgehog for Skyrme [13]. Indeed it is a non trivial fact that there are solutions to the constraints, like the special one found in [10] with the rational map, and the meromorphic ones for adjusted babyskyrme with an old trick due to Smirnov and Sobolev in [14].

Although there is a possibility to implement dressing, as mentioned in [四], the systematic method to construct solutions, which come naturally in $2 d$ from the affine algebraic structures, has still to be developed.

\section{Acknowledgments}

JSG would like to thank the La Plata colleagues for the perfect organization and all the participants for the friendly and rewarding atmosphere. This work is partially supported by grants from from CNPq (Brazil), NSF (PHY-9507829), EC (TMRERBFMRXCT960012) and DGICYT (PB 96-0960).

\section{References}

[1] Orlando Alvarez, Luiz A. Ferreira and J. Sánchez Guillén Nucl. Phys. B529 (1998) 689.

[2] D.I. Olive, N. Turok, Nucl. Phys. B220 (1983) 491, Nucl. Phys. B257 (1985) 277.

[3] L.A.Ferreira, J.L.Miramontes, J. Sánchez Guillén J. Math. Phys. 38 (1997) 882; J.L.Miramontes, hep-th/9809052.

[4] R.Donagi, E.Witten, Nucl. Phys. B460 (1996) 97; J. Edelstein and J.Mas, these Proceedings, hep-th/9902161.

[5] Ya. Aref'eva Theor. Mat. Phys. 43 (1980) 353, N.E.Bralic Phys. Rev. D22 (1980) 3090. 
[6] L.A. Ferreira and E. Leite, Integrable theories in any dimension and homogeneous spaces, hep-th/9810067, to appear in Nuclear Physics B.

[7] D. Gianzo, J.O. Madsen and J. Sánchez Guillén, Nucl. Phys. B537 (1999) 586.

[8] V.E. Zakharov and A.B. Shabat, Zh. Exp. Teor. Fiz. 61 (1971) 118-134; english transl. Soviet Phys. JETP 34 (1972) 62-69; P. Lax, Comm. Pure Appl. Math. 21 (1968) 467-490.

[9] P.G.O. Freund and R. Nepomechie, Nucl. Phys. B199 (1982) 482.

[10] H.Aratyn, L.A. Ferreira and A. Zimerman, Toroidal solitons in $3+1$ dimensional integrable theories, hep-th/9902141.

[11] J.M.F. Labastida, these proceedings.

[12] K. Fuji, Y. Homma and T. Suzuki,Phys. Lett. 438B (1998) 290

[13] J.O. Madsen and J. Sánchez Guillén, in preparation.

[14] K. Fuji, Y. Homma and T. Suzuki, hep-th/9809149. 